ORIGINAL ARTICLE

\title{
Empyema thoracis: a 10-year comparative review of hospitalised children from south Asia
}

\author{
A K Baranwal, M Singh, R K Marwaha, L Kumar
}

See end of article for authors' affiliations

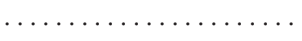

Correspondence to: Dr AKBaranwal, Department of Pediatrics, B.P. Koirala Institute of Health Sciences, Dharan, Nepal; baranwal1970@ yahoo.com

Accepted

21 February 2003

\begin{abstract}
Aims: To study the clinical and microbial profile of childhood empyema in South Asia and to identify the changes over the past three decades.

Methods: A total of 265 children (aged 1 month to 12 years) with empyema admitted to the Advanced Pediatric Center, PGIMER, Chandigarh, India in 1989-98, were reviewed retrospectively.

Results and Conclusions: One third of children were under 5. Culture positivity had decreased significantly $(48 \%$ v $75 \%)$ over the years. Staphylococcus aureus continues to be the commonest $(77 \%)$ aetiological agent; clustering was seen during hot and humid months (46\%). Culture positive Streptococcus pneumoniae cases also decreased $(9 \% \vee 27 \%)$; all were seen during the winter and spring season. Gram negative rods grew in more patients ( $11 \%$ v $7 \%$ ). Community acquired methicillin resistant $S$ aureus (MRSA) was isolated in three patients. Most children (93\%) were treated with parenteral cloxacillin and an aminoglycoside. Tube drainage (TD) was used in $92 \%$ of fibropurulent cases, and was successful in $79 \%$. Of 48 patients with failed TD, 12 needed decortication; limited thoracotomy was sufficient in the remaining 36. Surgery was mainly required by children with persistent pleural sepsis after 10 days of TD. Delaying surgery until 14 days had a significantly higher potential of requiring decortication. Early change to oral antibiotics (after 1-2 weeks of parenteral therapy) reduced the hospital stay significantly (17+7 v 23+7 days) without compromising long term outcome. Twenty two patients presenting late in the chronic stage underwent decortication at admission.
\end{abstract}

$\mathrm{P}$ leural empyema continues to be a serious problem despite recent advances in management. ${ }^{1}$ It is reported to occur in up to $28 \%$ children hospitalised for community acquired pneumonia. ${ }^{2}$ In developed countries the microbial profile has changed over the past five decades ${ }^{3}$ with increasing incidence of penicillin resistant Streptococcus pneumoniae $^{4}$ and methicillin resistant staphylococcus. ${ }^{5}$ However, the scenario is very different in developing countries, ${ }^{6-10}$ where empyema is associated with significant morbidity and consumption of scarce hospital resources. Optimal management in children is controversial, especially the duration of parenteral antibiotics and the role of surgery. Newer therapies, that is, fibrinolysis ${ }^{11}$ and video assisted thoracoscopic surgery ${ }^{12}$ have made the issue even more complex.

We present 10 years experience (1989-98) from a tertiary care centre in South Asia in order to provide evidence for the need to optimise the existing management guidelines and make them suitable for developing countries.

\section{MATERIALS AND METHODS}

The medical records of 265 children with empyema admitted in two general paediatric units (146 in LK's unit 1; 119 in RKM's unit 2) of the Advanced Pediatric Center at the Postgraduate Institute of Medical Education and Research, Chandigarh over a 10 year period (January 1989 to August 1998) were reviewed retrospectively. Aspiration of pus from the pleural space was the inclusion criterion; patients with tubercular empyema were excluded. The data extraction sheet included information on age, sex, presenting symptoms and signs, laboratory studies such as complete blood count, Gram stain and culture of pleural pus, blood culture, chest radiographs and ultrasonography. Culture of pus from other infected sites was also available. Of 265 patients, 243 (136 in unit 1, 107 in unit 2) were classified as having fibropurulent empyema ${ }^{13}$ and represented Light's class 5 to class 7 pleural effusion. ${ }^{14}$ After diagnostic thoracocentesis and obtaining appropriate cultures, parenteral antibiotics were started and a chest tube was inserted. Antibiotics were changed based on culture and sensitivity reports, or if clinical response was inadequate. Surgery was considered after failed tube drainage (TD). Surgery could usually be performed through a limited incision large enough to allow access to thoracic cavity (limited thoracotomy). However, patients with extensive pleural involvement underwent formal decortication. Twenty two patients (10 in unit 1, 12 in unit 2) had chronic organising empyema ${ }^{13}$ at admission- "peel" around the lung and signs of volume loss.

We analysed and compared policy regarding use of different therapeutic interventions (duration of parenteral therapy, trial of tube drainage, and timing of various surgical interventions used), and its impact on outcome (hospital stay, need of surgery, and improvement on follow up) in the two units (unitl $v$ unit 2). The data are compared with another study (1976-79) ${ }^{1}$ published from the same centre about 20 years ago. Statistical tests used were the $\mathrm{Z}$ test, Student's $t$ test, $\chi^{2}$ test with Yates's correction if needed, relative risk (RR), and odds ratio (OR) with $95 \%$ confidence interval $(\mathrm{CI})$.

\section{RESULTS}

\section{Clinical presentation}

There was no difference in demographic, clinical, and microbial characteristics between patients in unit 1 and unit 2. Table 1 shows clinical characteristics of patients with fibropurulent empyema; data from the previous study ${ }^{1}$ are

\footnotetext{
Abbreviations: $\mathrm{Cl}$, confidence interval; MRSA, methicillin resistant $S$ aureus; MSSA, methicillin sensitive $S$ aureus; $O R$, odds ratio; RR; relative risk; TD, tube drainage
} 
Table 1 Comparison of clinical features of patients with fibropurulent empyema seen during the two study periods

\begin{tabular}{lll}
\hline & $1989-98(\mathbf{n}=\mathbf{2 4 3})$ & $1976-79(\mathbf{n}=\mathbf{7 5})$ \\
\hline Sex (M:F) & $2.4: 1$ & $2.9: 1$ \\
Age (years) & $57(23.5 \%)$ & $12(16 \%)$ \\
$<1$ & $105(43 \%)$ & $37(49 \%)$ \\
$1-5$ & $81(33 \%)$ & $26(35 \%)$ \\
$>5$ & $94(39 \%)$ & - \\
Nutritional status (weight - \% of 50th centile of Harvard Standard) & $11(47 \%)$ & - \\
$>80 \%$ (normal) & $35(14 \%)^{*}$ & $20(27 \%)^{*}$ \\
$80-60 \%$ & $77(32 \%)$ & $27(36 \%)$ \\
$<60 \%$ & $54(23 \%)$ & $16(21 \%)$ \\
Duration of fever (n=239) & $63(26 \%)^{*}$ & $11(15 \%)^{*}$ \\
$<7$ days & $165(68 \%)$ & $40(53 \%)$ \\
$8-14$ days & $66(27 \%)$ & $34(45.5 \%)$ \\
$15-21$ days & $12(5 \%)$ & $1(1.5 \%)$ \\
Intrathoracic pathology & $131(54 \%)$ & - \\
Right sided empyema & $21(9 \%)$ & $13(17 \%)$ \\
Left sided empyema & $65(27 \%)$ & - \\
Bilateral empyema & $22(9 \%)$ & $3(4 \%)$ \\
Consolidation & $10(16 \%)$ & - \\
Pneumatoceles & $17(7 \%)$ & - \\
Pneumothorax & & \\
Bronchopleural fistula & & \\
Treatment received prior to referral & & \\
Parenteral antibiotics only & & \\
Parenteral antibiotics and tube thoracostomy & & \\
\hline * $p<0.05$. & &
\end{tabular}

also given for comparison. Duration of fever varied from 1 to 60 days. Most of them (91\%) had received oral antibiotics for fever and/or pneumonia before admission; 57 (24\%) had received parenteral therapy. Only a few received specific antistaphylococcal (cloxacillin) and anti-streptococcal (benzylpenicillin) antibiotics. Seventeen children underwent TD prior to admission. Nine patients developed empyema in the hospital as a complication of pneumonia. Three had septic shock at admission, while one developed it later. Thirty four ( $14 \%$ ) patients (20 in unit 1 and 14 in unit 2) had empyema as part of disseminated disease with two or more noncontiguous organ involvement and positive microbial evidence in the form of Gram stain and/or culture. They had subcutaneous abscess $(n=13)$, pyopericardium $(n=11)$, pyomyositis $(\mathrm{n}=3)$, liver abscess $(\mathrm{n}=3)$, meningitis $(n=2)$, acute suppurative otitis media $(n=2)$, intra-abdominal sepsis $(n=2)$, septic arthritis $(n=2)$, or osteomyelitis $(n=1)$. Patients with a longer duration of symptoms ( $>15$ days) had a higher incidence of anaemia (haemoglobin $<110 \mathrm{~g} / \mathrm{l})\left(\chi^{2}=5.78, \mathrm{p}<0.02\right)$ and severe anaemia (haemoglobin $<70 \mathrm{~g} / \mathrm{l})\left(\chi^{2}=6.23, \mathrm{p}<0.05\right)$. Total leucocyte count was raised in $45 \%$ of patients. Four patients, including three infants had neutropenia (absolute neutrophil count $<1500 / \mathrm{mm}^{3}$ ).

\section{Predisposing conditions}

A preceding history of pustules was present in 31 patients $(13 \%)$. Some had preceding blunt trauma to the chest $(n=4)$ or viral infections (chickenpox in one and measles in three). The incidence of measles decreased significantly from $11 \%$ $(8 / 75)^{1}$ to $1.2 \%(3 / 243) \quad(\mathrm{p}<0.001)$ during the past two decades. Contemporary reports from areas with poor immunisation coverage still have measles as a significant predisposing factor. ${ }^{810}$ Sixty two per cent of patients were malnourished. Severe malnutrition (weight below $60 \%$ of the 50 th centile of the Harvard standard $)^{15}$ was seen in only $14 \%$ of cases compared to $27 \%(\mathrm{p}<0.05)$ in the previous study. ${ }^{1}$ Empyema occurred as a result of contiguous extension from subphrenic and amoebic liver abscesses in one case each.

\section{Microbiological characteristics}

Bacterial aetiology was confirmed in 117 patients from whom 121 isolates were obtained (table 2). A total of 113 children
(97\%) grew one microorganism. Another organism grew in four patients in addition to $S$ aureus: Escherichia coli in three and Haemophilus influenzae in one. The organisms were obtained from various body fluids-pleural pus $(n=100)$, blood $(n=13)$, subcutaneous abscess $(n=7)$, liver abscess $(\mathrm{n}=2)$, pyomyositis $(\mathrm{n}=1)$, and cerebrospinal fluid $(\mathrm{n}=1)$. In one patient, pleural pus revealed Entamoeba histolytica.

Staphylococcus spp. were the commonest isolates (90/121). Eighty five $(94 \%)$ of them were coagulase positive and methicillin sensitive (MSSA); two were coagulase negative staphylococcus. Methicillin resistant $S$ aureus (MRSA) grew in three patients without any known risk factor. ${ }^{16}$ Culture negative patients having "clusters" of Gram positive cocci in pus smear $(n=13)$, active and/or healing pustules $(n=9)$, pneumothoraces $(n=42)$, and pneumatoceles $(n=8)$ were also managed with anti-staphylococcal therapy. ${ }^{17}$ Thus staphylococcus was considered to be the infecting organism in $162(67 \%)$ patients. It predominated in all the age groups, more so among the under $5 \mathrm{~s}$ (table 2 ). The majority of $S$ pneumoniae were isolated from older children. Most (11/14) of the Gram negative rods were also seen among older children, who were ill for longer (22.8 (11.9), 3-60 days) than those with staphylococcus (12.7 (9.4), 1-45 days), S pneumoniae (18.4 (17.4), 3-30 days), and anaerobes (15.0 (7.0), 1020 days).

On comparing these microbial findings with those of the previous study, ${ }^{1}$ organisms were cultured in fewer patients $(48 \% \vee 75 \%, \mathrm{p}<0.001)$ with a higher yield of staphylococcus $(76 \% \vee 56 \%, \mathrm{p}<0.01)$. In contrast, a threefold decline $(9 \% v$ $27 \%, \mathrm{p}<0.01)$ occurred in culture positive $S$ pneumoniae disease. The yield of Gram-negative rods increased ( $11 \% v$ $7 \%, \mathrm{p}>0.05)$. The antibiotic sensitivity pattern revealed the appearance of community acquired MRSA; however, none had penicillin resistant $S$ pneumoniae.

\section{Annual and seasonal distribution}

The number of empyema cases was similar every year. About half $(47 \%)$ of them presented during the hot and humid months (May to August) of the year (goodness of fit $\left.\chi^{2}=20.1, p<0.001\right)$. This was due to clustering of staphylococcus positive cases (46\% in May to August, goodness of fit $\chi^{2}=6.1, \mathrm{p}<0.02$ ). In contrast, all $S$ pneumoniae positive cases 
Table 2 Distribution of microorganisms by age in patients with fibropurulent empyema ( $n=243 ; 121^{*}$ isolates from 117 patients)

\begin{tabular}{|c|c|c|c|c|c|}
\hline \multirow[b]{2}{*}{ Pathogens } & \multicolumn{4}{|l|}{ Age groups } & \multirow[b]{2}{*}{ Total } \\
\hline & $\begin{array}{l}<1 \text { year } \\
(n=57)\end{array}$ & $\begin{array}{l}0-4 \text { years } \\
(n=150)\end{array}$ & $\begin{array}{l}5-9 \text { years } \\
(n=62)\end{array}$ & $\begin{array}{l}\geqslant 10 \text { years } \\
(n=31)\end{array}$ & \\
\hline Staphylococcus & $21(37 \%) \dagger$ & $61(41 \%)$ & $18(29 \%)$ & 11 (35.5\%) & $90 / 121$ \\
\hline Gram negative rods & $3(5 \%)$ & $3(2 \%)$ & $8(13 \%)$ & $3(10 \%)$ & $14 / 121$ \\
\hline Pneumococcus & 1 & $3(2 \%)$ & $2(3 \%)$ & $5(16 \%)$ & $10 / 121$ \\
\hline$S$ pyogenes & 0 & 1 & 0 & 0 & 1 \\
\hline$E$ fecalis & 0 & 0 & 1 & 1 & 2 \\
\hline$H$ influenzae & 1 & 1 & 1 & 0 & 2 \\
\hline Anaerobes & 0 & 1 & 1 & 0 & 2 \\
\hline
\end{tabular}

were seen during the winter and spring months (November to April).

\section{Antimicrobial therapy}

A combination of intravenous cloxacillin $(200 \mathrm{mg} / \mathrm{kg} / \mathrm{day}$, every six hours) and an aminoglycoside was initiated empirically in 226 patients (128/136 in unit $1,98 / 107$ in unit 2 ). The remainder of the patients received benzylpenicillin (2-4 lac unit/kg/day, every six hours) in addition. Antibiotics were altered depending on the clinical response and/or sensitivity report.

There was one difference in the antibiotic policy between the two units. Unit 1 patients received parenteral cloxacillin for a longer period (3.0 (0.8) weeks; range 2-6 weeks). In unit 2, antibiotics were given orally once active infection was under control, evidenced by subsidence of fever and respiratory distress, improvement in general condition, and cessation of pus discharge, usually after 1-4 weeks $(2.2$ (0.8) weeks). Seven children developed a drug rash to cloxacillin; ciprofloxacin was then used for an equivalent period. Staphylococci are largely sensitive to ciprofloxacin in our hospital and other hospitals of the region. ${ }^{18}$ Culture reports did not affect the duration of parenteral therapy; however, patients with associated pyopericardium, septic arthritis, and osteomyelitis were given parenteral antibiotics for longer (3-4 weeks). Benzylpenicillin was used in 44 children for 1-4 weeks. Ten of them grew $S$ pneumoniae and received benzylpenicillin monotherapy for 19 days on average (range 10-24 days) followed by oral amoxicillin to complete 4-6 weeks therapy.

\section{Closed tube drainage}

Most patients (223 of 243, 92\%) underwent closed TD (table 3), which was successful in 175 patients (overall success rate $78 \%$ ). Nine patients needed TD on both sides for bilateral empyema. The tube was usually inserted ( 212 of 232 tubes, 91\%) on the day of admission. TD was tried for a variable period and was highly dependent on the attending physician. In the group of patients who responded to conservative therapy alone (178 tubes in 175 patients), 41 had their tubes removed by the fifth day and 92 patients by the tenth day. On average, unit 2 patients had tubes removed earlier (8.6 (6.0) $v 10.4$ (5.9) days, $\mathrm{p}<0.05$ ); and fewer patients $(8 \% \vee 19 \%, \mathrm{p}<0.05)$ had TD for more than 14 days. Chest tubes failed in $48(22 \%)$ patients (table 3$)$.

During 1989-91, six children were discharged with an open tube after failure of closed TD. They had a longer (28.8 (13.2) days) hospital stay compared to those who underwent surgery in addition to procedure related morbidity even after discharge. Repeated thoracocentesis was performed in only two children, and both needed surgery. Tube insertion was
Table 3 Need for various therapeutic interventions in patients with fibropurulent empyema

\begin{tabular}{llll}
\hline $\begin{array}{l}\text { Therapeutic } \\
\text { interventions }\end{array}$ & $\begin{array}{l}\text { Unit } \mathbf{1} \\
(\mathbf{n}=136)\end{array}$ & $\begin{array}{l}\text { Unit 2 } \\
(\mathbf{n}=107)\end{array}$ & $\begin{array}{l}\text { Total } \\
(\mathbf{n}=\mathbf{2 4 3})\end{array}$ \\
\hline Initial TD & $125 / 136(92 \%)$ & $101 / 107(94 \%)$ & $223 / 243(92 \%)$ \\
Failed TD & $22 / 122(18 \%)$ & $26 / 101(26 \%)$ & $48 / 223(22 \%)$ \\
Limited thoracotomy & $17 / 22(77 \%)$ & $19 / 26(73 \%)$ & $36 / 48(75 \%)$ \\
Decortication & $5 / 22(23 \%)$ & $7 / 26(27 \%)$ & $12 / 48(25 \%)$ \\
\hline TD, tube drainage.
\end{tabular}

deferred in 20 patients as only minimal pus could be obtained on aspiration; they responded to antibiotics alone.

\section{Surgical management}

Surgery was performed in 48 (22\%) children (table 3) primarily for uncontrolled infection: persistent fever, respiratory distress or pus discharge, large loculations, organised collection; some required it for restrictive pleural thickening or bronchopleural fistula. Continuing pus discharge was the single most important factor associated with surgery, which was twice as likely if a child had not improved with TD in 10 days (RR $1.86,95 \%$ CI 1.13 to 3.04). A third of patients (32\%) with pus discharge beyond 10 days needed surgery.

Limited thoracotomy with breakdown of loculations and drainage of the pleural space was performed in 36 patients, while formal decortication was needed in 12 . Limited thoracotomy was sufficient in the majority of those who underwent surgery during the second week. Decortication was needed mostly if surgery was delayed beyond it. The patient was six times more likely to need a formal decortication if he had over 14 days of TD without good effect (OR 5.8, 95\% CI 1.41 to 23.85). Patients requiring surgery for uncontrolled infection became afebrile within 23 days and the chest tube could be removed after 4-5 days. All had full expansion of the lungs. The majority went home 7-10 days after surgery. Surgery was deferred in 30 children despite evidence of organising loculation, pleural thickening, or both on imaging, as they were asymptomatic and had reasonably good lung expansion.

\section{Duration of hospitalisation}

Table 4 shows the hospital stay in patients requiring different treatments. Patients requiring tube drainage alone stayed in hospital for a shorter period in unit 2 compared to unit 1 $(p<0.01)$. Unit 2 patients stayed in hospital on average one week more for limited thoracotomy $(p<0.001)$ and two weeks more for decortication $(\mathrm{p}<0.001)$. Such a difference was not observed in unit 1 as all the patients received longer 
parenteral antibiotic treatment (4-6 weeks) irrespective of clinical response. Patients with disseminated infection remained in hospital for a longer period (23 (7) days), especially if they had pyopericardium (30 (6) days) or septic arthritis (38 (17) days).

\section{Outcome}

Among fibropurulent cases, 239 of 243 survived; only four patients died ( $1.6 \%$ mortality). Two patients died of tension pneumothorax. One succumbed to uncontrolled septicaemia and another to postoperative shock. Three patients left the hospital against medical advice due to financial constraints. On follow up, all the children were doing well. None had clinically significant restrictive disease.

\section{Chronic organising empyema}

Twenty two children (median age 6 years) were referred in the organising stage. They had been ill for a mean of 58.0 (24.5) days (range 15-75 days). Eleven patients were mild to moderately malnourished, while severe malnutrition was seen in three (14\%). Staphylococcus was obtained from four patients (blood, one; pleural pus, three), one being methicillin resistant. Pleural aspirate grew Gram negative rods in six patients (Klebsiella pneumoniae in one, Pseudomonas aeruginosa in five) and anaerobic bacteria in one. These patients had evidence of volume loss and/or thick pleural peel and underwent decortication as their first procedure. All were well on clinical follow up.

\section{DISCUSSION}

\section{Microbiology of empyema}

The clinical presentation of childhood empyema had changed little, however some changes were noticed in the microbiology over the past three decades. Yield on culture was significantly lower $(48 \%)$ compared to the previous study ${ }^{1}$ and other reports from developing countries, ${ }^{6-10}$ which varied from $57 \%$ to $82 \%$. It could be due to prior use of antibiotics and late referral ${ }^{19}$ more children $(26 \% \vee 15 \%, \mathrm{p}<0.05)$ reported after two weeks of fever compared to the previous study. ${ }^{1}$

Staphylococcus continues to be the commonest causative organism in the tropics and subtropics. ${ }^{6-10}$ Patients were seen mostly during the hot and humid months. In the tropical zone, excessive sweating and moist skin favour growth of cutaneous flora, leading to a high incidence of staphylococcal pyoderma. ${ }^{20}$ Haematogenous spread from these lesions may lead to pneumonia and parapneumonic empyema. ${ }^{17}$ Others have also reported a proportion of patients with pyodermal lesions. ${ }^{18}$ Considering the importance of staphylococcus in causation of parapneumonic empyema in tropical areas, an anti-staphylococcal agent should be included in first-line treatment of severe pneumonia.

Over the 10 year period, three children grew MRSA. Though hospital acquired MRSA is increasingly being seen in the region, ${ }^{21}$ community acquired MRSA has not been reported in children until now. It is feared that dissemination of MRSA may be occurring in the community due to widespread use of $\beta$ lactam antibiotics. ${ }^{22}$ Emergence of community acquired MRSA is a cause for concern and needs further investigation.

A significant decline in $S$ pneumoniae positive cases has been seen over the past two decades, probably because of prior antibiotic therapy. Penicillin resistant $S$ pneumoniae were not seen. The incidence of Gram negative rods has increased. These organisms are usually important during the first six months of life and are reportedly not seen after five years. ${ }^{23}$ However, in the present study, most of patients were above 5 years of age. The majority of these isolates are likely to be superadded infections. Anaerobes are recognised as important cause of childhood empyema in the west; ${ }^{24}$ studies from developing countries have not specifically looked for these. ${ }^{16-10}$ Further investigation is required to define the exact role of anaerobes in childhood empyema from these countries.

\section{Management}

Management of primary empyema continues to be controversial in terms of duration of antibiotic therapy and the indications for and timing of surgery.

\section{Medical therapy}

Specific anti-staphylococcal antibiotics should be initiated empirically in tropical and subtropical countries, considering the predominance of staphylococcus. We used high dose cloxacillin $(200 \mathrm{mg} / \mathrm{kg} /$ day $)$. It is important to use antistreptococcal antibiotics (benzylpenicillin) as well until the culture reports are available. Aminoglycosides are used for their synergistic effect and may be given for the first 5-7 days.

Duration of antibiotic therapy depends on pathogens, response to therapy, and complications. However, information on optimal duration of parenteral therapy is lacking. ${ }^{25}$ In unit 2, antibiotics were administered orally after patients became afebrile, respiratory distress subsided, and significant loculations were ruled out, usually after 7-14 days. This approach reduced the hospital stay and the cost of care without affecting clinical outcome. Oral antibiotics should be continued for a further 2-4 weeks.

\section{Pleural space drainage}

The pleural space should be drained for lung re-expansion, relief of respiratory distress, and to prevent formation of thick pleural peel. Thoracocentesis is likely to be inadequate for patients presenting late in the fibropurulent stage, ${ }^{26}$ and continuous closed TD usually leads to clinical and radiological improvement within 24 hours. The tube should be removed once drainage becomes minimal $(<10$ to $15 \mathrm{ml} /$ day), ${ }^{3}$ irrespective of roentgenographic resolution. A maximum of 7-10 days trial should be pursued. Most children respond to conservative management ${ }^{27} 28$ and pulmonary function tests are normal by 18 months despite significant initial abnormality. ${ }^{27}$ It was successful in $78 \%$ of our cases.

Table 4 Comparison of hospital-stay (days) in the two units, in relation to various modalities used

\begin{tabular}{lllll}
\hline & Successful TD & Limited thoracotomy & Decortication & p value \\
\hline Unit 1 $(n=122)$ & $23.2(7.4)(n=100)$ & $24.7(7.4)(n=17)$ & $27.7(7.9)(n=5)$ & $p>0.05^{*}$ \\
Unit 2 $(n=101)$ & $17.2(7.2)(n=75)$ & $23.8(4.7)(n=19)$ & $36.4(21.9)(n=7)$ & $p<0.001^{*}$ \\
p value & $p<0.01$ & $p>0.05$ & $p>0.05$ & \\
\hline
\end{tabular}

$T D$, tube drainage

*For difference between successful TD and limited thoracotomy, and difference between successful TD and decortication. 


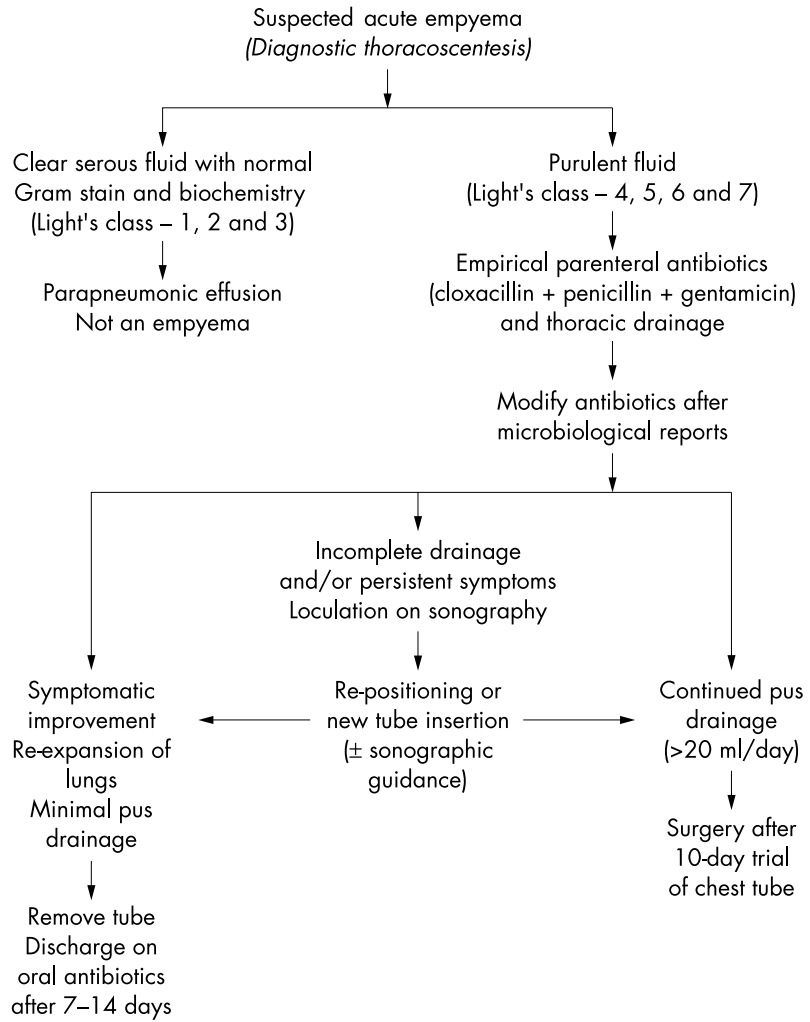

Figure 1 Proposed protocol for management of childhood empyema in developing economies.

\section{Role of surgery}

Some authors recommend an aggressive surgical approach, ${ }^{29}$ others are more conservative. ${ }^{27}$ Complete pleural drainage is rarely necessary in children and radiographs usually clear by three months in most cases. ${ }^{27}$ Thus until controlled trials prove the superior safety and effectiveness of an aggressive surgical protocol vis-à-vis the conservative approach, surgery is advised only for persistent pleural sepsis. ${ }^{3}$

If TD fails after a 10 day trial, surgery is indicated for debridement of the pleural space. Fibroblastic invasion usually starts after two weeks of disease, ${ }^{30}$ causing an adherent fibrous peel. Thus surgery soon after 10 days of failed TD is likely to be a relatively minor procedure with shorter hospital stay. For empyema in the organising phase, full thoracotomy with decortication remains the treatment of choice. Decortication was needed in $12(5 \%)$ of our patients. Intrapleural instillation of fibrinolytic drugs has been used and proven to be effective, ${ }^{11}$ but high cost prohibits its routine use in developing countries.

\section{Hospital stay}

Prolonged hospital stay used to be the rule for children with empyema in the past, especially for staphylococcal cases. ${ }^{13} 25$ Even in a recent study, patients stayed in hospital for more than three weeks, ${ }^{31}$ similar to our unit 1 patients. In contrast, the unit 2 patients who responded to conservative therapy alone, stayed in hospital for 17 days on average (table 4), the difference being attributable to early switch over to oral therapy. Such an approach led to reductions in hospital stay, its associated morbidity, and the overall cost of care, without compromising the outcome.

\section{CONCLUSIONS}

Staphylococcus continues to be the most important organism for childhood empyema in South Asia. These were mostly
MSSA; few were community acquired MRSA. Lower yield on culture was probably due to prior use of antibiotics. Late referral may be responsible for superadded infections with Gram negative rods. The role of anaerobes needs to be defined. The use of anti-staphylococcal and anti-streptococcal antibiotics is the mainstay of empirical therapy. Conservative management with TD was adequate in most cases. Switching over to oral antibiotics is satisfactory and cost effective alternative if signs of active infection have subsided. Surgery is indicated for uncontrolled pleural sepsis even after 10 days of TD. A few may require formal decortication, especially if surgery is delayed beyond 14 days. Asymptomatic patients with residual radiological features do not require surgery. The long term prognosis is excellent.

The proposed protocol (fig 1 ) is simple and can be followed safely in almost all secondary health care centres. It is intended for optimal utilisation of scarce facilities available in developing countries.

\section{Authors' affiliations}

A K Baranwal, Departments of Pediatrics, B.P. Koirala Institute of Health Sciences, Dharan, Nepal

M Singh, R K Marwaha, L Kumar, Postgraduate Institute of Medical

Education and Research, Chandigarh, India

\section{REFERENCES}

1 Kumar L, Gupta AP, Mitra S, et al. Profile of childhood empyema thoracis in North India. Indian J Med Res 1980;72:854-9.

2 Byington CL, Spencer LY, Johnson TA, et al. An epidemiological investigation of a sustained high rate of pediatric parapneumonic empyema: risk factors and microbiological associations. Clin Infect Dis 2002;34:434-40.

3 Givan DC, Eigen H. Common pleural effusions in children. Clin Chest Med 1998; 19:363-71.

4 Hardie WD, Roberts NE, Reising SF, et al. Complicated parapneumonic effusions in children caused by penicillin-nonsusceptible Streptococcus pneumoniae. Pediatrics 1998;101:388-92.

5 Musher DM, Lamm N, Darouiche RO, et al. The current spectrum of Staphylococcus aureus infection in a tertiary care hospital. Medicine 1994;73:186-208.

6 Maziah W, Choo KE, Ray JG, et al. Empyema thoracis in hospitalised children in Kelantan, Malaysia. J Trop Pediatr 1995;41:185-8.

7 Mangete EDO, Kombo BB, Legg-Jack TE. Thoracic empyema: a study of 56 patients. Arch Dis Child 1993;69:587-8.

8 Mishra OP, Das BK, Jain AK, et al. Clinico-bacteriological study of empyema thoracis in children. J Trop Pediatr 1993;39:380-1.

9 Asindi AA, Efem SE, Asuquo ME. Clinical and bacteriological study on childhood empyema in south eastern Nigeria. East Afr Med J 1992;69:78-82.

10 Ghosh S, Chakraborty CK, Chatterjee BD. Clinico-bacteriological study of empyema thoracis in infants and children. J Indian Med Assoc 1990:88:189-90.

11 Thomson $\mathbf{A H}$, Hull J, Kumar MR, et al. Randomised trial of intrapleural urokinase in the treatment of childhood empyema. Thorax 2002;57:343-7.

12 Grewal H, Jackson RJ, Wagner CW, et al. Early video-assisted thoracic surgery in the management of empyema. Pediatrics 1999;103:e63.

13 Chan W, Keyser-Gauvin E, Davis GM, et al. Empyema thoracis in children: a 26-year review of the Montreal Children's Hospital experience. J Pediatr Surg 1997;32:870-2.

14 Light RW, Rodriguez RM. Management of parapneumonic effusions. Clin Chest Med 1998;19:373-82.

15 Nutrition Subcommittee of the Indian Academy of Pediatrics. Report. Indian Pediatr 1972;9:360.

16 Gorak EJ, Yamada SM, Brow JD. Community-acquired methicillin-resistant Staphylococcus aureus in hospitalised adults and children without known risk factors. Clin Infect Dis 1999;29:797-800.

17 Goel A, Bamford L, Hanslo D, et al. Primary staphylococcal pneumonia in young children: a review of 100 cases. J Trop Pediatr 1999:45:233-6.

18 Karthikeyan G, Premkumar K. Neonatal sepsis: Staphylococcus aureus as the predominant pathogen. Indian J Pediatr 2001;68:715-17.

19 Cham CW, Haq SM, Rahamim J. Empyema thoracis: a problem with late referral? Thorax 1993:48:925-7.

20 Singh G. Heat, humidity and pyoderma. Dermatologica 1973;147:342-7.

21 Verma S, Joshi S, Chitnis V, et al. Growing problem of methicillin resistant staphylococci-Indian scenario. Indian J Med Sci 2000;54:535-40.

22 Salaria M. Singh M. Methicillin resistant-Staphylococcus aureus. Indian Pediatr 2001;38:29-36.

23 Patwari AK, Bisht S, Srinivasan A, et al. Aetiology of pneumonia in hospitalised children. J Trop Pediatr 1996;42(1):15-20.

24 Brook I. Microbiology of empyema in children and adolescents. Pediatrics 1990:85:722-6. 
25 Freii BJ, Kusmies $\mathrm{H}$, Nelson JD, et al. Parapneumonic effusions and empyema in hospitalised children: a retrospective review of 227 cases. Pediatr Infect Dis $J$ 1984;3:578-91.

26 LeMense GP. Strange C, Sahn SA. Empyema thoracis: therapeutic management and outcome. Chest 1995;107:1532-7.

27 Gocmen A, Kiper N, Toppare $M$, et al. Conservative treatment of empyema in children. Respiration 1993;60:182-5.

28 Hull J, Thomson A. Empyema thoracis: a role for open thoracotomy and decortication. Arch Dis Child 1999:80:581.
29 Kosloske AM, Cartwright KC. The controversial role of decortication in the management of pediatric empyema. J Thorac Cardiovasc Surg 1988;96:166-70.

30 Kennedy AS, Agness M, Bailey L, et al. Decortication for childhood empyema-the primary provider's peccadillo. Arch Surg 1991;126:1287-91.

31 Shoseyov D, Bibi H, Shatzberg G, et al. Short-term course and outcome of treatments of pleural empyema in pediatric patients: repeated ultrasoundguided needle thoracocentesis vs. chest tube drainage. Chest 2002;121:836-40. 